\title{
A SHORT-TERM ASSESSMENT OF ROUTINE CHEMISTRY PARAMETERS BY SIGMA METRICS AND QUALITY GOAL INDEX RATIO IN A TERTIARY CARE HOSPITAL LABORATORY
}

\author{
Salma Sayeed ${ }^{1}$, Suresh Babu Ganji², Ramadevi Mopuri ${ }^{3}$ \\ 1 Postgraduate Student, Department of Biochemistry, Osmania Medical College, Hyderabad, Telangana, India. \\ ${ }^{2}$ Associate Professor, Department of Biochemistry, MNJIO\&RCC and Osmania Medical College, Hyderabad, Telangana, India. \\ 3 Professor and HOD, Department of Biochemistry, Osmania Medical College, Hyderabad, Telangana, India.
}

\section{ABSTRACT}

\section{BACKGROUND}

Sigma metrics is an important tool to evaluate the errors in quality control of laboratory system. It can easily quantify the exact number of errors by combining bias, precision, and total allowable error (TEa). Quality goal index is a newer parameter to represent the relative extent to which both bias and precision meet their respective quality goals. The aim of the present study is to assess the Internal and External quality control by Sigma Metrics and Quality Goal Index ratio (QGI).

\section{METHODS}

This observational study was conducted at Clinical Biochemistry Laboratory, Osmania General Hospital, Hyderabad, Telangana. Data of 8 most commonly run analytes: glucose, urea, creatinine, total proteins, total bilirubin, cholesterol, albumin and uric acid were extracted over a period of 3 months. The two levels of controls of these parameters were run on a Beckman Coulter AU5800 autoanalyser. Mean, SD, and coefficient of variance (CV) were calculated for each month from IQC. Bias obtained from EQAS. Sigma, QGI and TE were calculated subsequently.

\section{RESULTS}

The sigma metrics for level 1 for all eight parameters indicated that 5 failed to meet a minimum sigma quality performance with metrics less than 3 and another 2 just met minimal acceptable performance with sigma metrics between 3 and 6 . For level 2, none of the parameters achieved six sigma, of which 5 parameters had metrics less than 3 and 3 had metrics between 3 and 6 . The calculated TE of all the parameters was less than the specified TEa. QGI ratio indicated that out of five parameters of level 1 and level 2, which failed to meet six sigma quality performances, the main problem was imprecision in all parameters except album in which showed both imprecision and inaccuracy for both levels.

\section{CONCLUSIONS}

Both Internal and External quality control for the most routinely run parameters in our hospital need stringent monitoring in order to fulfill the requirements of a standard clinical laboratory.

\section{KEY WORDS}

External Quality Assurance Scheme (EQAS), Internal Quality Control (IQC), Quality Goal Index (QGI), Six Sigma

HOW TO CITE THIS ARTICLE: Sayeed S, Ganji SB, Mopuri R. A short-term assessment of routine chemistry parameters by sigma metrics and quality goal index ratio in a tertiary care hospital laboratory. J. Evolution Med. Dent. Sci. 2019;8(29):2303-2306, DOI: $10.14260 /$ jemds/2019/505

\section{BACKGROUND}

Clinical biochemistry investigations are important in diagnosis and treatment. It is essential to follow a proper quality management system (QMS) to provide accurate and reliable reports in an agreed upon time frame. Sigma metrics is an important tool to evaluate the errors in quality control of laboratory system. Sigma is a metric that quantifies the performance of a process at a rate of defects per million. The sigma value indicates how often errors are likely to occur. The sigma value of one corresponds to $31 \%$ of accuracy which is equal to 698,000 defects per million tests.

'Financial or Other Competing Interest': None.

Submission 28-05-2019, Peer Review 04-07-2019,

Acceptance 10-07-2019, Published 22-07-2019.

Corresponding Author:

Dr. Suresh Babu Ganji,

Associate Professor,

Department of Biochemistry,

MNJIO\&RCC and Osmania Medical College,

Hyderabad, Telangana, India.

E-mail: sushwasa@gmail.com

DOI: $10.14260 /$ jemds $/ 2019 / 505$
A total defects per million of 66,807 , have $93.3 \%$ accuracy giving a 3 sigma and to achieve $99.9997 \%$ of accuracy, 3.4 defects per million are allowed which is a six sigma metric and this value is a high standard set for any laboratory.[1] Thus it is evident that the higher the sigma value, the lower the chance of false test results by the laboratory. It can easily quantify the exact number of errors by combining bias, precision, and total allowable error (TEa ) a sigma level $<3$ is an indication of a poor performance procedure, whilst a good performance is indicated by a sigma level $>3$. Sigma level of 6 or greater indicates world class performance.[2] To calculate precision and bias, internal quality control (IQC) and external quality assurance scheme (EQAS) are being carried out in our laboratory. IQC is run as per NABL guidelines and is interpreted by Levy Jennings chart and Westgard rules. Quality goal index is a newer parameter to represent the relative extent to which both bias and precision meet their respective quality goals.

The aim of the present study is to assess the Internal and External quality control by popular tool, Sigma Metrics and Quality Goal Index ratio (QGI) (a newer tool). 


\section{METHODS}

This observational study was conducted at clinical biochemistry laboratory of Osmania general hospital, Hyderabad, Telangana, which provides laboratory service to a more than 1000 bed tertiary care hospital. Data was extracted over a period of 3 months from July to September 2018. A total of 8 most commonly run analytes were included in the study which were: glucose, urea, creatinine, total proteins, total bilirubin, cholesterol, albumin and uric acid. All parameters were run along with IQC and EQAS. IQC data was analysed for imprecision and EQAS data for inaccuracy. The two levels of controls of these parameters were run on a Beckman Coulter AU5800 auto analyser by using Randox kits obtained from the manufacturer following the standard operating procedures at our lab. As per laboratory policy, two levels of controls (level 1: normal and level 2; pathological, Randox laboratories limited) were run twice daily along with monthly EQAS samples from Christian medical college, Vellore as a routine. The laboratory follows the Westgard rule to accept and reject the run. $13 \mathrm{~s}, 22 \mathrm{~s}, \mathrm{R} 4 \mathrm{~s}, 41 \mathrm{~s}$, and $10 \mathrm{x}$ were considered a rejection, and $12 \mathrm{~s}$ as a warning rule to each respective run. Mean, SD, and coefficient of variance (CV) were calculated for each month for both levels.

\section{Sigma Metrics}

Mean of the CV of both levels and bias was calculated and used for estimating sigma metrics by the following formula. Sigma $=($ TE a- Bias $) /$ CV.

The Minimum Acceptable Performance of Process was a 3 Sigma Level

\section{Quality Goal Index}

QGI represents the relative extent to which both bias and precision meet their respective quality goals. It was calculated using the following formula;

QGI= Bias $/ 1.5 \mathrm{CV}$

QGI $<0.8$ indicates imprecision, $>1.2$ indicates inaccuracy, and QGI score 0.8 to 1.2 indicates both imprecision and inaccuracy. ${ }^{[3-5]}$

\section{Coefficient of Variation}

The coefficient of variation (CV) is the SD expressed as a percentage and is a measure of the variability of an assay. [6] It indicates the imprecision.

$\mathrm{CV}=(\mathrm{SD} /$ mean $) \times 100$

\section{BIAS}

Bias is the systematic difference between the expected results obtained by the laboratory tests method and the results that would be obtained from an accepted reference method. It indicates the accuracy of the results. The difference or deviation of the lab value from the mean of peer group values of same method is reported as the Bias \% in the EQAS report, monthly.

\section{TEa}

Allowable Total error, TEa was followed as per the Clinical Laboratory Improvement Amendments (CLIA) guidelines. TEa can also be taken from various sources of guidelines like for example; RCPA, Ricos, EUBIVAS and others. We can also adopt various TEa for different parameters to improve the tightness of the ranges which depends on the lab QC policy.
Total error (TE) of parameters was also calculated by the following formula $[7,8]$. TE= BIAS+ $1.65 \mathrm{CV}$ This value should be within the adopted TEa range.

\section{RESULTS}

\begin{tabular}{|c|c|c|c|c|}
\hline Parameter & July & August & September & Average \pm SD \\
\hline Glucose & 2.99 & 2.76 & 3.66 & $3.13 \pm 0.46$ \\
\hline Urea & 3.10 & 4.77 & 4.20 & $4.02 \pm 0.84$ \\
\hline Creatinine & 4.07 & 4.57 & 12.96 & $7.2 \pm 0.1$ \\
\hline Total bilirubin & 19.86 & 3.59 & 6.31 & $9.92 \pm 8.7$ \\
\hline Total protein & 3.42 & 3.01 & 4.51 & $3.64 \pm 0.8$ \\
\hline Albumin & 7.14 & 12.27 & 10.26 & $9.89 \pm 2.6$ \\
\hline Cholesterol & 5.31 & 9.21 & 7.44 & $7.32 \pm 1.9$ \\
\hline Uric acid & 2.09 & 4.01 & 2.48 & $2.86 \pm 1.0$ \\
\hline $\begin{array}{l}\text { Table 1. The CV\% of the 8 Parameters of Level 1 Internal Quality } \\
\text { Control for a Period of 3 Months (July to September 2018) and Their } \\
\text { Average }\end{array}$ \\
\hline
\end{tabular}

\begin{tabular}{|c|c|c|c|c|}
\hline Parameter & July & August & September & Average \pm SD \\
\hline Glucose & 3.53 & 2.69 & 2.57 & $2.93 \pm 0.5$ \\
\hline Urea & 4.63 & 4.58 & 4.21 & $4.47 \pm 0.2$ \\
\hline Creatinine & 4.36 & 4.72 & 13.43 & $7.5 \pm 5.13$ \\
\hline Total bilirubin & 3.04 & 2.84 & 12.04 & $5.97 \pm 5.25$ \\
\hline Total protein & 4.30 & 3.05 & 4.34 & $3.89 \pm 0.73$ \\
\hline Albumin & 6.71 & 9.83 & 6.66 & $7.73 \pm 1.8$ \\
\hline Cholesterol & 8.40 & 10.85 & 2.60 & $7.28 \pm 4.23$ \\
\hline Uric acid & 4.53 & 12.31 & 2.28 & $6.37 \pm 5.3$ \\
\hline Table 2. The CV \% of the 8 Parameters of Level 2 Internal Quality \\
Control for a Period of 3 Months (July to September 2018) and Their \\
Average \\
\hline
\end{tabular}

\begin{tabular}{|c|c|c|c|c|}
\hline Parameter & July & August & September & average \pm SD \\
\hline Glucose & -6.7 & -4.9 & -4.8 & $-5.4 \pm 0.77$ \\
\hline Urea & -4.2 & 3.9 & 1.6 & $0.43 \pm 4.17$ \\
\hline Creatinine & -5.3 & 3.3 & 0.0 & $-0.66 \pm 4.3$ \\
\hline Total bilirubin & 0.0 & 4.3 & 10.0 & $4.76 \pm 5.0$ \\
\hline Total protein & -12.0 & -6.1 & -14.0 & $-10.7 \pm 4.1$ \\
\hline Albumin & -16.0 & -3.3 & -16.7 & $-12 \pm 7.5$ \\
\hline Cholesterol & -7.2 & 0.4 & -5.0 & $-3.93 \pm 3.9$ \\
\hline Uric acid & -7.2 & -21.9 & -24.4 & $-17.8 \pm 9.3$ \\
\hline
\end{tabular}

Table 3. The Bias \% Obtained from External Quality Assurance Scheme from CMC Vellore for 8 Parameters for a Period of 3 Months (July to September 2018) and Their Average

\begin{tabular}{|c|c|c|c|c|c|}
\hline Parameter & TEa & TE (L1) & TE (L2) & Sigma Level 1 & Sigma Level 2 \\
\hline Glucose & 10 & -0.23 & -0.56 & 4.92 & 5.25 \\
\hline Urea & 9 & 7.06 & 7.80 & 2.13 & 1.91 \\
\hline Creatinine & 15 & 11.22 & 11.71 & 2.17 & 2.08 \\
\hline Total bilirubin & 20 & 21.12 & 14.61 & 1.53 & 2.55 \\
\hline Total protein & 10 & -4.69 & -4.28 & 5.68 & 5.32 \\
\hline Albumin & 10 & 4.31 & 0.75 & 2.22 & 2.84 \\
\hline Cholesterol & 10 & 8.14 & 8.08 & 1.90 & 1.91 \\
\hline Uric acid & 17 & -13.11 & -7.31 & 12.17 & 5.46 \\
\hline
\end{tabular}

Table 4. Sigma Metrics (Level 1 and Level 2) and Quality Goal Index Ratio (Level 1 and Level 2) of 8 Parameters Calculated from Coefficien of Variation (Level 1 and Level 2), Total Allowable Error and Bias \% for a Period of 3 Months

\begin{tabular}{|c|c|c|}
\hline Sigma Metrics & Level 1 & Level 2 \\
\hline$<3$ & $\begin{array}{c}\text { Urea, creatinine, Total bilirubin, } \\
\text { cholesterol, albumin }\end{array}$ & $\begin{array}{c}\text { Urea, creatinine, Total bilirubin, } \\
\text { cholesterol, albumin }\end{array}$ \\
\hline $3-6$ & Glucose, total protein & Glucose, total protein, uric acid \\
\hline$>6$ & Uric acid & - \\
\hline \multicolumn{3}{|c|}{ Table 5. Sigma Metrics of Various Parameters } \\
\hline
\end{tabular}

\begin{tabular}{|c|c|c|c|c|}
\hline Parameter & Qc Levels & Sigma & QGI & Problem \\
\hline \multirow{2}{*}{ Urea } & Level 1 & 2.13 & 0.07 & Imprecision \\
& Level 2 & 1.91 & 0.06 & Imprecision \\
\hline \multirow{2}{*}{ Creatinine } & Level 1 & 2.17 & 0.06 & Imprecision \\
& Level 2 & 2.08 & 0.05 & Imprecision \\
\hline \multirow{2}{*}{ Total bilirubin } & Level 1 & 1.53 & 0.31 & Imprecision \\
& Level 2 & 2.55 & 0.53 & Imprecision \\
\hline \multirow{2}{*}{ Cholesterol } & Level 1 & 1.90 & 0.35 & Imprecision \\
& Level 2 & 1.91 & 0.35 & Imprecision \\
\hline \multirow{3}{*}{ Albumin } & Level 1 & 2.22 & \multirow{2}{*}{0.809} & Imprecision \\
& & & & Inaccuracy \\
& Level 2 & 2.84 & \multirow{2}{*}{1.03} & Imprecision \\
& & & Inaccuracy \\
\hline
\end{tabular}

Table 6. The Sigma Metrics and Quality Goal Index Ratio for The Five Parameters with Low Sigma Metrics (<3) for Both Levels 


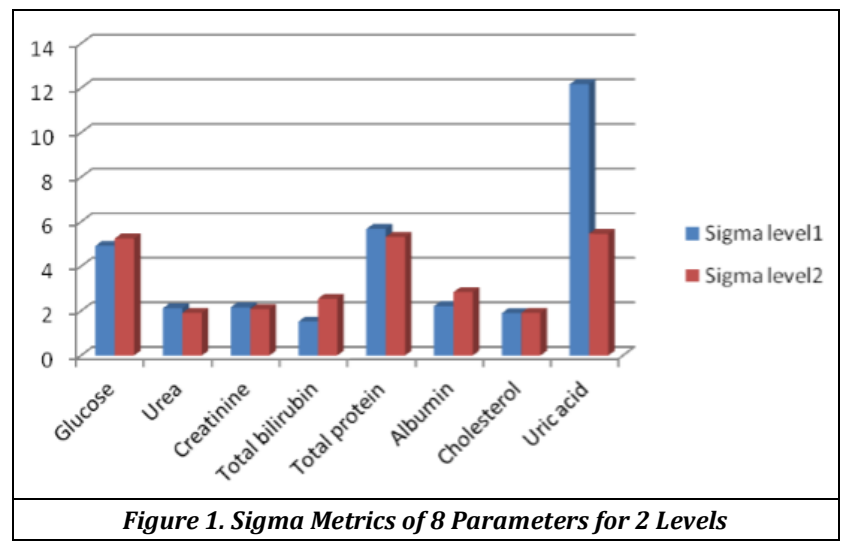

Table 5 shows Sigma metrics of various parameters for two levels of controls. The sigma metrics for the level 1 indicated that 7 of the 8 parameters fell short of meeting six sigma quality performances. Of these, 5 failed to meet a minimum sigma quality performance with metrics less than 3 and another 2 just met minimal acceptable performance with sigma metrics between 3 and 6 . For level 2, the data collected indicated 8 of the 8 parameters did not achieve six sigma quality performances, out of which 5 had metrics less than 3 and 3 had metrics between 3 and 6 . The calculated TEa of all the parameters was less than the specified TEa (CLIA).

Table 6 summarises the QGI ratio of analytes with lower sigma values $(<3)$. QGI ratio indicated that out of five parameters of level 1 and level 2, which failed to meet six sigma quality performances, the main problem was imprecision in all parameters except albumin which showed both imprecision and inaccuracy for both levels.

\section{DISCUSSION}

IQC ensures a continuous watch of the analytical system, so as to check whether the results are reliable and can be reported. EQAS involves analysing and reporting of the control samples sent by an external agency, at a predefined time interval which in Clinical chemistry parameters are tested once in a month. The Bias \% is obtained from the EQAS report; means how far our result has shifted from the peer group mean value. The exact number of errors done by the laboratory in the analytical phase cannot be assessed by running internal and external QCs, but can be quantified by Sigma metrics. ${ }^{[9]}$ In this three-months-study, only Uric acid showed a sigma of $>6$ for level 1 and sigma between 3 and 6 for level 2 and IQC showing excellent precision for level 1 but not for L2. Urea, creatinine, total bilirubin, cholesterol, albumin were short of sigma metrics with a sigma of $<3$ for both levels of 1 and 2 . Glucose and total protein showed sigma between 3 and 6 for level 1 and 2. In our study, CV\% was based on a typical 90-day study. We get a robust CV with a long-term QC data, e.g., over a 6-month or 1-year period. Using a precision value calculated for a shorter period of time could result in a more optimistic estimate and result in a higher Sigma metric.[10] The variations in sigma values for few analytes between our study and others can be due to the difference in the methodology, Traceability calibrators used, instrument used, quality control material used, and other preanalytical and analytical conditions. ${ }^{[4]}$ We calculated the QGI for parameters with sigma $<3$ at both concentrations of quality control products, noted differences in QGI corresponding to different sigma values, and determined priority measures for quality improvement. When QGI less than 0.8 , imprecision was bigger hence Urea, Creatinine, Total bilirubin and Cholesterol precision is to improve and When QGI was higher than 0.8 to 1.2 , here for the Albumin, the bias was also relatively large, suggesting that the priority should be to improve precision as well as accuracy. Clinical laboratories should regularly measure detection performance for all parameters and develop individualized quality control plans for all analytes to provide long-term, feasible measures for continuous improvement of assay quality.[11] Verma et al study reveals that if TE of an analyte is within allowable error limits specific for that analyte, bias \% and CV \% might be more reliable than sigma metrics. ${ }^{[12]}$ TE for levels $1 \& 2$ shows the values did not exceed the allowable total error recommended by CLIA. The suggested guidelines for choosing the Westgard rules and levels of IQC processed are as follows: for the biochemical parameters with Sigma Scale 6 or above (Excellent performance), evaluate with one level of QC per day (Alternating levels between days) and follow 1-3 s Westgard rule alone. With Sigma Scale 4-6 (Good/acceptable performance), evaluate with two levels of control once daily and follow 1-3 s, 2-2 s, R4 s Westgard multirules. With Sigma Scale 3-4 (Poor performance), use two levels of controls twice daily and follow 1-3 s, 2-2 s, R4s, and 4-1 s Westgard multirules. With Sigma Scale of $<3$ (problem analyte), root cause analysis should be performed; method performance must be improved before the method can be routinely used.[13]

A limitation of this study is we did not include a long-term $\mathrm{CV}$ and bias. Periodic calculation of Sigma metrics is appropriate to determine if assay quality has been maintained, has decreased, or has improved. Sigma metrics thus represent another quality assurance tool to be monitored periodically to assess changes in assay quality or else it will only reflect assay performance at the time the data was collected and thus represent a "snapshot". Naturally, performance can change over time for a variety of reasons (e.g., reagent lot to lot variation) [10]. There are certain limitations in clinical application of sigma metrics for few analytes like hormones, for which IQC-CV\% and EQAS-Bias\% prove to be more reliable than sigma, provided they are within the total allowable error limits of CLIA.

\section{CONCLUSIONS}

Sigma metric analysis provides a benchmark for the laboratory to design a protocol for IQC, address poor assay performance, and assess the reliability as well as the efficiency of existing laboratory process. It warrants a root cause analysis from the past towards future improvement. Internal and External quality control are essential to monitor quality in a stringent manner for the most routinely run parameters in any laboratory. Hence, it is very much necessary to follow the Westgard rules to improve the precision and accuracy to the betterment of sigma metrics along with the tool, QGI.

\section{ACKNOWLEDGEMENTS}

The authors acknowledge the laboratory technical staff for their cooperation and assistance. 


\section{REFERENCES}

[1] Nevalainen D, Berte L, Kraft C, et al. Evaluating laboratory performance on quality indicators with the six sigma scale. Arch Pathol Lab Med 2000;124(4):516-9.

[2] Adiga US, Preethika A, Swathi K. Sigma metrics in clinical chemistry laboratory - a guide to quality control. Al Ameen J Med Sci 2015;8(4):281-7.

[3] Westgard JO, Westhard SA. The quality of laboratory testing today: an assessment of sigma metrics for analytic quality using performance data from proficiency testing surveys and the CLIA criteria for acceptable performance. Am J Clin Pathol 2006;125(3):343-54.

[4] Kumar BV, Mohan T. Sigma metrics as a tool for evaluating the performance of internal quality control in a clinical chemistry laboratory. J Lab Physicians 2018;10(2):194-9.

[5] Plebani M. Errors in clinical laboratories or errors in laboratory medicine? Clin Chem Lab Med 2006;44(6):750-9.

[6] Sciacovelli L, O'Kane M, Skaik YA, et al. Quality indicators in laboratory medicine: from theory to practice. Preliminary data from the IFCC working group project "Laboratory Errors and Patient Safety". Clin Chem Lab Med 2011;49(5):835-44.
[7] Cao S, Qin X. Application of Sigma metrics in assessing the clinical performance of verified versus nonverified reagents for routine biochemical analytes. Biochem Med 2018;28(2):020709.

[8] Venkatesh T. Accreditation requirement of laboratory medicine in India. Indian $\mathrm{J}$ Clin Biochem 2008;23(3):207-8.

[9] Westgard JO. Internal quality control: planning and implementation strategies. Ann Clin Biochem 2003;40(Pt 6):593-611.

[10] Hens K, Berth M, Armbruster D, et al. Sigma metrics used to assess analytical quality of clinical chemistry assays: importance of the allowable total error (TEa) target. Clin Chem Lab Med 2014;52(7):973-80.

[11] Liu Q, Fu M, Yang F, et al. Application of Six Sigma for evaluating the analytical quality of tumor marker assays. J Clin Lab Anal 2019;33(2):e22682.

[12] Verma M, Dahiya K, Ghalaut VS, et al. Assessment of quality control system by sigma metrics and quality goal index ratio: a roadmap towards preparation for NABL. World J Methodol 2018;8(3):44-50.

[13] Westgard JO. Six sigma quality design and control. $2^{\text {nd }}$ edn. Madison, WI: Westgard QC Inc., 2006. 